\title{
Clasificación inmunohistoquímica del cáncer de mama y su importancia en el diagnóstico, pronóstico y enfoque terapéutico
}

Immunohistochemical Classification of Breast Cancer and its Importance in the Diagnosis, Prognosis and Therapeutic Approach Classificação de imunocitoquímica de câncer de mama e suaimportância no diagnóstico, prognóstico e abordagemterapêutica

\author{
Sergio Andrés Melo-Sánchez, MD., Esp. * \\ Leidy Tatiana Gelvez-Parra, MD. ** \\ Sonia Esperanza Osma-Zambrano, MD., Esp., MSc. ***
}

\begin{abstract}
Resumen
Introducción: El cáncer de mama es un problema de salud pública creciente, representa un desafío para los sistemas de salud. Durante el año 2012 se registraron en el mundo 1.6 millones de casos nuevos de cáncer de mama lo cual corresponde al $25.0 \%$ de todos los casos en la población femenina. Es una enfermedad multifactorial, con gran variedad clínica y diversos tipos histológicos. La inmunohistoquímica se utiliza para clasificar el tumor, evaluar el pronóstico, mejorar el diagnóstico y orientar el manejo de las neoplasias mamarias. Objetivo: Revisar la literatura actual relacionada con la clasificación inmunohistoquímica del cáncer de mama con énfasis en diagnóstico, pronóstico y manejo. Metodología: Este artículo de revisión se realizó con 50 artículos recuperados, resultado de una estrategia de búsqueda completa de publicaciones a partir del año 2000. Resultados: Los principales marcadores biológicos utilizados con fines pronósticos o predictivos son: el grado histológico, la positividad de receptores de estrógeno (ER),
\end{abstract}

progesterona (PR) y la presencia del oncogén Her2/neu; estos 3 últimos fundamentan la clasificación inmunohistoquímica actual. La expresión de estas moléculas denomina las lesiones tumorales, siendo los tumores luminales aquellos que expresan ER o PR y tumores no luminales los que carecen de dichos receptores. En estos subtipos se han descrito y demostrado diferencias en la respuesta a las diversas modalidades terapéuticas. Conclusiones: La clasificación inmunohistoquímica del cáncer de mama permite individualizar cada caso y juega un papel fundamental en el diagnóstico, pronóstico y la selección de una terapéutica oncológica específica más efectiva. [Melo-Sánchez SA, Gelvez-Parra LT, OsmaZambrano SE. Clasificación inmunohistoquímica del cáncer de mama y su importancia en el diagnóstico, pronóstico y enfoque terapéutico. MedUNAB 2016; 18(3): 193-203].

Palabras clave: Neoplasias de la Mama; Inmunohisto química; Receptores estrogénicos; Receptores de progesterona; Neoplasias de la mama triple negativas.

* Médico, Especialista en Epidemiologia. Coordinador de Estudios Clínicos de la Unidad de Investigación y Educación, Instituto del Corazón de Bucaramanga, Colombia.

** Médica general, Especialista en salud ocupacional y riesgos laborales (C), Vidamedical IPS, Colombia

*** Médica Ginecobstetra, Magister en Epidemiología, Docente Facultad de Medicina, Universidad Autónoma de Bucaramanga, Santander, Colombia.

Autor de correspondencia: Sergio Andrés Melo Sánchez, Instituto del Corazón Bucaramanga, Colombia. Correo electrónico: sergioam51391@gmail.com. 


\section{Abstract}

Introduction: Breast cancer is a growing public health problem and it represents a challenge for health systems.In 2012, in the world, 1.6 million new cases of breast cancer which correspond to $25.0 \%$ of all cases in the female population were registered. It is a multifactorial disease with great clinical variety and diverse histological types.Immunohistochemistry is used to classify the tumor, assess the prognosis, improve the diagnosis and guide the management of breast neoplasias. Objective: To review the current literature regarding the immunohistochemical classification of breast cancer with emphasis on diagnosis, prognosis and management of it. Methodology: This review article was performed with 50 articles retrieved, as a result of a comprehensive search strategy of publications from 2000. Outcomes: The main biomarkers used for prognostic or predictive purposes are: histological grade, estrogenreceptor-positive (ER), progesterone (PR) and the presence of oncogene Her2/neu; being these last 3 the foundation of the current immunohistochemical classification.The expression of these molecules is used to refer to tumor lesions; luminal tumors are those that express ER or PR and, not luminal tumors those that lack such receptors.In these subtypes have described and demonstrated differences in response to various therapeutic modalities.Conclusions: Immunohistochemical classification of breast cancer allowspersonalizing each case and plays a key role in the diagnosis, prognosis and selection of a more effective specific oncological therapy. [Melo-Sánchez SA, Gelvez-Parra LT, Osma-Zambrano SE. Immunohistochemical Classification of Breast Cancer and its Importance in the Diagnosis, Prognosis and Therapeutic Approach. MedUNAB 2016; 18(3): 193-203].

Keywords: Breast Neoplasias; Immunohistochemistry; Receptors, Estrogen; Receptors; Progesterone; Triple Negative Breast Neoplasias.

\section{Introducción}

El cáncer de mama es la neoplasia maligna más común entre las mujeres y continúa siendo la más frecuente si se analizan ambos sexos. Según las cifras de GLOBOCAN 2012, se registraron 1.6 millones de casos incidentes que corresponde al $25.0 \%$ de todos los casos en la población femenina. A nivel global, el cáncer de glándula mamaria, es la quinta causa de muerte entre todos los cánceres, ocasionando un total de 52,000 defunciones. Representa la primera causa de mortalidad en los países menos desarrollados con 324,000 defunciones, (14.3\% del total) y la segunda en los países con mayores recursos con 198,000 defunciones, (15.4\% del total) precedida por el cáncer de pulmón (1). Para ese mismo año, en todo el continente americano se registraron 256,222 casos nuevos (29.6\%) y 48,850 muertes $(14.9 \%)$ por cáncer de mama. El panorama en Latinoamérica y el Caribe fue 152,059 casos nuevos $(27.0 \%)$ y 43,208 muertes $(14.9 \%)$ y específicamente en Sur América fueron 115,881 casos $(28.2 \%)$ y un total de $32,014(15.3 \%)$ muertes (1).

\section{Resumo}

Introdução: O câncer da mama é um problema crescente de saúde pública, representa umdesafio para os sistemas de saúde. Em 2012 foram registrados no mundo 1,6 milhões de novos casos de câncer da mama, correspondente a $25,0 \%$ de todos os casos na populaçãofeminina. É umadoençamúltifatorial, com grande variedade clínica e diversos tipos histológicos. A imuno-histoquímica foi utilizada para classificar o tumor, avaliar o prognóstico, melhorar o diagnóstico e orientar o manejo de neoplasias mamárias. Objetivo: Revisar a literatura atual sobre a classificaçãoimuno-histoquímica de câncer de mama, comênfase no diagnóstico, prognóstico e gestão. Metodologia: Este artigo de revisãofoi realizado com 50 artigos recuperados, o resultado de umaestratégia de pesquisa abrangente de publicações a partir de 2000 . Resultados: Os principaisbiomarcadores utilizados para fins de prognósticoouprevisãosão: grau histológico, receptor de estrogênio positivo (ER), progesterona (PR) e a presença de oncogên Her2/neu; sendo que a classificaçãoimunohistoquímica se baseia nos 3 últimos. A expressãodestas moléculas designa o tipo das lesõestumorais, os tumores luminaissãoaquelas que expressam ER e PR tumores nãoluminais que nãopossuemtais receptores. Nestes subtipos se encontra escrito e demonstrado que existe diferença na respostaàsvárias modalidades terapêuticas. Conclusões: A classificaçãoimuno-histoquímica do câncer da mama pode individualizar cada caso e desempenhaum papel fundamental no diagnóstico, prognóstico e seleçãomais eficaz de tratamento oncológico específico e maisefetivo. [Melo-Sánchez SA, Gelvez-Parra LT, OsmaZambrano SE. Classificação de imunocitoquímica de câncer de mama e suaimportância no diagnóstico, prognóstico e abordagemterapêutica. MedUNAB 2016; 18(3):193-203].

Palavras-chave: Neoplasias da Mama; Imuno-Histoquímica; Receptores Estrogênicos; Receptores de Progesterona; Neoplasias de Mama Triplo Negativas.

El comportamiento epidemiológico en América Latina es hacia el aumento rápido y progresivo evidenciado en los indicadores de incidencia y mortalidad $(2,3)$, siendo la más alta en Bahamas, seguida de Uruguay. En cuanto a mortalidad, son Bahamas, Trinidad y Tobago y Uruguay los países que presentan mayores tasas; lo cual posiblemente refleja fallas en el acceso a intervenciones de detección temprana y manejo (2).

La situación colombiana es preocupante; en el 2012 se registraron 8,686 casos nuevos de cáncer de mama lo que corresponde a un $23.4 \%$ de todas las lesiones tumorales, explicó 2,649 muertes $(7.0 \%)$ y se estimaron 31,340 casos prevalentes a 5 años (18.3\%) (1). El Registro Poblacional de Cáncer del área metropolitana de Bucaramanga (RCPAMB) para el periodo 2003-2007 estableció en la población femenina del área metropolitana de Bucaramanga (AMB) que el cáncer de mama fue la neoplasia diagnosticada con mayor frecuencia y se constituyó en la primera causa de muerte, correspondiendo al $13.1 \%$ de todas las muertes, seguido por el cáncer de 
pulmón y estómago (4), estimó en el quinquenio 2001-2005 una tasa de mortalidad ajustada por edad de 17.5 casos por 100.000 mujeres, y una tasa de incidencia ajustada por edad de 37.9 casos por 100.000 mujeres la cual superaba el estimado nacional a esa fecha (5).

En los países desarrollados se ha visto una disminución de la mortalidad por cáncer de mama, resultado de un diagnóstico y manejo más temprano y oportuno (2); sin embargo, a nivel nacional continúa siendo un problema de salud pública $(1,2,4-6)$.

Es una enfermedad multifactorial y heterogénea, sus expresiones clínicas son diversas así que el diagnóstico es un reto para la salud individual y colectiva, planteándose la necesidad de establecer un pronóstico basado en el análisis minucioso de parámetros clínicos, anatomopatológicos y biológicos $(6,7)$. En el consenso de expertos de St Gallen 2015 se comparó la inmunohistoquímica con el estándar de oro, la clasificación molecular basada en expresión genética resultando esta última técnicamente más compleja y poco disponible por razones logísticas o financieras; así que actualmente la clasificación inmunohistoquimica resulta ser clínicamente significativa, técnicamente simple, reproducible, y fácilmente disponible para resolver esta problemática (8).

La clasificación inmunohistoquimica se fundamenta en la identificación de marcadores celulares, utilizados con fines pronósticos o predictivos. La positividad de los receptores de estrógeno (ER), progesterona (PR) y la presencia del oncogén del factor de crecimiento de tipo epidérmico humano 2 (Her $2 /$ ne) definen y fundamentan esta clasificación. La expresión de estos marcadores determina el tipo tumoral y el fenotipo al que pertenecen (luminal A o B, HER2 positivo y triple negativo); de esta manera se define el diagnóstico y se orienta el abordaje terapéutico en cada caso brindando una terapéutica oncológica específica más efectiva, evitando el uso de esquemas de tratamientos no adecuados; y permitiendo cumplir con la necesidad de individualización, integralidad y oportunidad en el manejo $(6,7)$.

Este trabajo tiene como objetivo hacer una revisión bibliográfica en relación con los fenotipos celulares propuestos por la clasificación inmunohistoquímica del cáncer de mama, haciendo énfasis en las características y criterios para el diagnóstico, frecuencia de presentación, respuesta terapéutica y pronóstico según los subtipos, entre otros factores.

\section{Metodología}

Se realizó una búsqueda sistemática en las bases de datos de Pubmed, Ovid, Scielo, Lilacs, utilizando los términos descriptores de ciencias de la salud "Medical Subject Headings (MeSH)": "Breast Neoplasms", "Neoplasms by Histologic Type”, "Immunohistochemistry/classification”,
"Histological Techniques/classification", "Prognosis", "Female", "Adult", "Young adult", "middleaged", "Receptors, Estrogen”, "Receptors, Progesterone”, "Triple Negative Breast Neoplasms". Y los términos no MeSH: "Subtyping of breast cancer", "Histological analysis", "Histologic grade", "Neoplasms epidemiology”, "Oncogene Protein HER-2”, "Immunohistochemical classification", "Immunohistochemistry breast cancer", "Prognosis cancer”.

Inicialmente se realizó una búsqueda utilizando el operador booleano $O R$ para cada uno de los bloques temáticos de interés, y posterior a la selección de material útil se llevó a cabo una búsqueda más específica utilizando los operadores $A N D$ y NOT. Terminada la exploración se filtraron los resultados por título y resumen logrando un total de 75 artículos, los cuales fueron sometidos a lectura crítica vigilando que cumplieran con criterios mínimos de calidad y de inclusión considerados para esta revisión.

Se incluyeron trabajos observacionales retrospectivos y prospectivos, ensayos clínicos controlados, revisiones de la literatura y metaanálisis que hayan aplicado, evaluado y comparado la clasificación inmunohistoquímica del cáncer de mama según las denominaciones internacionales vigentes; solo se tuvieron en cuenta trabajos publicados desde el año 2000 a la fecha, e igualmente se incluyeron reportes de organizaciones de reconocimiento mundial líderes en el tema. Se aplicaron límites de edad (mayores de edad), género (población femenina) e idioma (artículos en inglés y español). Se excluyeron trabajos realizados con lesiones tumorales que no tuvieron origen primario en la glándula mamaria.

Del total de la búsqueda bibliográfica finalmente se utilizaron 50 artículos para la redacción de este trabajo. Una de las limitaciones de esta revisión es la dificultad en la recuperación de literatura médica, y que solo incluyó artículos en inglés y español pero consideramos que se elaboró con los artículos más relevantes y con buena calidad metodológica.

\section{Bases de la clasificación inmunohisto- química del cáncer de mama}

La tendencia internacional en relación a la clasificación de los carcinomas mamarios, es superar las debilidades predictivas y pronósticas del método tradicional de clasificación basado en la histopatología $(7,9)$, utilizando la inmunohistoquímica y estableciendo la clasificación celular a partir de la expresión de receptores hormonales de membrana (estrógeno "ER", progesterona "PR", HER $2 /$ neu) y proteínas como citoqueratinas basales (CKs 5 o 6), proteína $\mathrm{P} 63$ y receptor del factor de crecimiento epidérmico (EGFR) $(7,8)$. La expresión de estas proteínas receptoras establece su clasificación en tumores luminales 


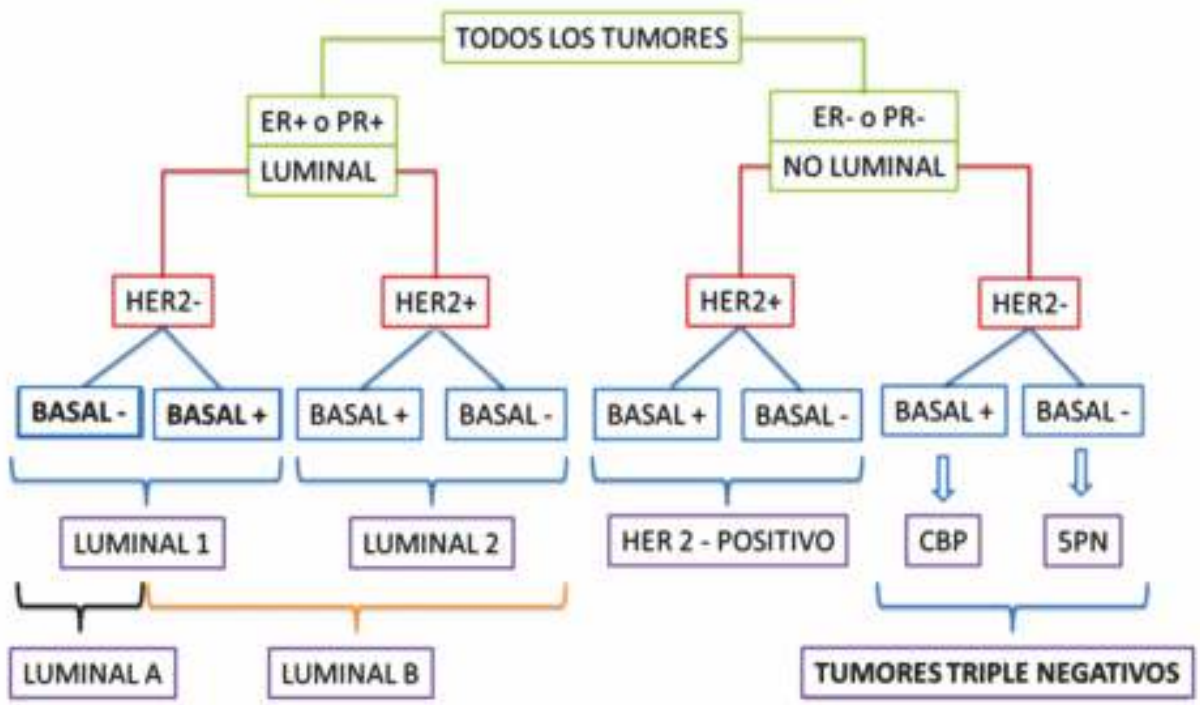

Figura 1. Esquema de la clasificación inmunohistoquímica del cáncer de mama.

Fuente: Tomado y adaptado de: Blows F, Driver K, Schmidt M, Broeks A, Leeuwen F; Subtyping of Breast Cancer by Immunohistochemistry to Investigate a Relationship between Subtype and Short and Long Term Survival: A Collaborative Analysis of Data for 10,159 Cases from 12 Studies. PloS Medicine; May 2010.

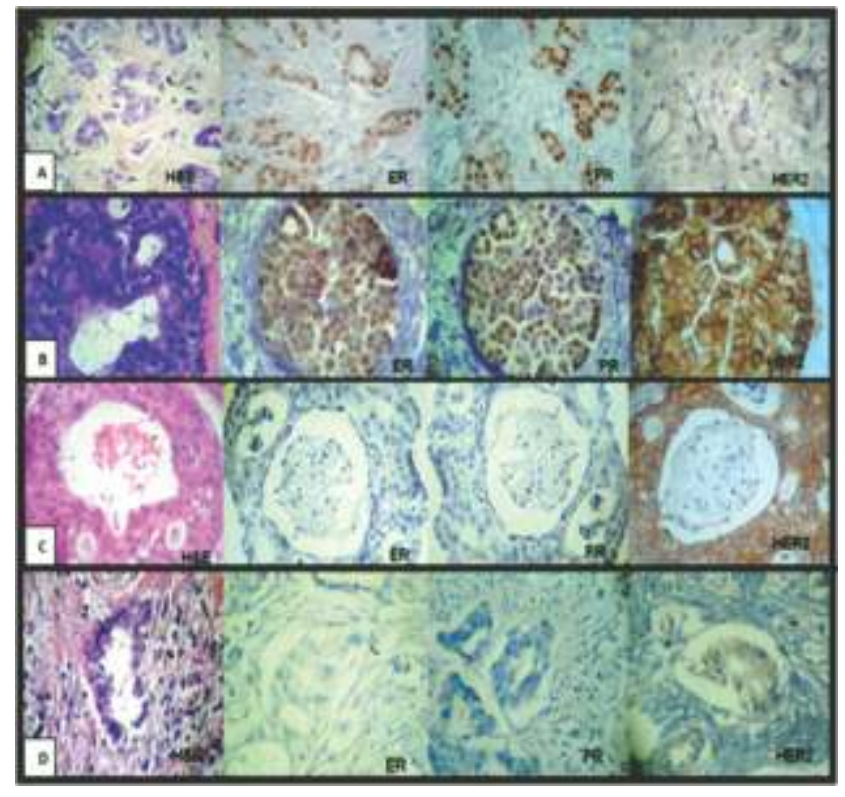

Figura 2. Grados de inmunohistoquimica marcados por hematoxilina y eosina e Inmunohistoquímica: A. Luminal A (receptores de estrógeno "ER" +, receptores de progesterona positivos "PR" +, receptor para el factor de crecimiento de tipo epidérmico humano "HER2" -). B. Luminal B (ER+, PR+, HER2+). C. HER2+ (ER-, PR-, HER2+). D. Triple negativo (ER-, PR-, HER2-). Fuente: Ana Laura Calderón-Garcidueñas, MD, PhD, Gabriela Martínez-Reyes MD, Adriana I. Gallardo-Gómez Chem., Augusto Rojas-Martínez, Ricardo M. Cerda-Flores; Triple Marker Immunohistochemestry Analysis in Breast Cancer Mexican Patients; Patología Revista latinoamericana Volumen 50, núm. 2, abril-junio, 2012. aquellos que expresan ER o PR y no luminales los que carecen de la expresión de dichos receptores. La importancia radica en que estos subtipos tumorales descritos muestran diferencias significativas en la respuesta a las diversas modalidades terapéuticas (Figura 1 y Figura 2) $(6-8,10)$.

Dependiendo de la expresión del receptor HER2, las lesiones tumorales del grupo luminal se diferencian en otros dos subtipos, siendo los tumores luminales 1 los que expresan los receptores hormonales y no expresan el HER2; mientras que los tumores luminales 2 son aquellos tumores positivos tanto para receptores hormonales como para HER2 $(7,8)$. Adicionalmente; se pueden evaluar en ambos subgrupos otras moléculas como citoqueratinas basales (CK5/6) o EGFR entre otros $(7,8)$. Con el fin de dar mayor aplicación terapéutica se puede obtener una nueva sub-clasificación (7-9), denominando luminal A, aquellos tumores luminal 1 sin expresión de moléculas basales, y como luminal B, aquellos tumores positivos para receptores hormonales que expresan o no HER2 y moléculas basales $(6-8,10)$.

Los tumores no luminales son los negativos para receptores hormonales (ER y PR). A su vez, estos se subdividen según la expresión de HER2, definiéndose tumores HER2 positivos, si expresan dichas moléculas y tumores triple negativos a los que no las expresen (TNP: nombrado así por la ausencia de los tres marcadores claves en la clasificación, ER, PR, y HER2) $(7,8,10)$. Esta última categoría de TNP es dividida en dos subcategorías según el tipo de moléculas basales que expone, denominándose como tumores del grupo basal nuclear (CBP) aquellos que expresan una de las 
Tabla 1. Sistema de puntuación ALLRED para la evaluación de receptores de estrógenos y progesterona.

\begin{tabular}{cccc}
\hline Proporción del score & $\%$ células positivas & Proporción de tinción & Intensidad de tinción \\
\hline 0 & 0 & 0 & Ninguna \\
\hline 1 & $<1 \%$ & 1 & Débil \\
\hline 2 & $1 \%$ a $10 \%$ & 2 & Intermedio \\
\hline 3 & $11 \%$ a $33 \%$ & 3 & Fuerte \\
\hline 4 & $34 \%$ a $66 \%$ & Interpretación \\
\hline 5 & $>67 \%$ & Negativo \\
\hline La puntuación de proporción y puntuación de intensidad se suman para una puntuación total \\
\hline \multicolumn{3}{c}{ Score total } \\
\multicolumn{3}{c}{$\mathbf{0 , 2}$} \\
\hline $3, \mathbf{4}, \mathbf{6 , 7 , 8}$
\end{tabular}

Fuente: Tomado y adaptado de Lester S, Bose S, Chen Y, Connolly J, De Baca M, Fitzgibbons P, et al. Protocol for the Examination of Specimens From Patients With Invasive Carcinoma of the Breast, Protocol applies to all invasive carcinomas of the breast, including ductal carcinoma in situ (DCIS) with microinvasion; Based on AJCC/UICC TNM, 7th edition; 2012.

citoqueratinas basales principales (CK5, CK6, CK14) o EGFR positivo (6-8), o recibiendo el nombre de tumores del fenotipo 5 negativo (5PN) pues carecen de marcación celular(ER, PR, HER2, CK5/6 Y EGFR) (7).

\section{Consenso para la medición y clasificación inmunohistoquímica de las lesiones por cáncer de mama}

El Colegio Americano de Patología (CAP) y la Asociación Americana de Oncología clínica (ASCO) establecen presencia negativa de receptores hormonales (ER, PR) cuando en la valoración de inmunohistoquímica la expresión de estos receptores en las células tumorales es inferior al $1.0 \%$, la existencia de receptores entre el $1 \%$ y el $10 \%$ es considerada una de las tinciones débilmente positivas, mientras que un resultado superior al $10 \%$ representa un valor francamente positivo; en los casos determinados como débilmente positivos se debe realizar una estricta evaluación del riesgo/beneficio de la endocrinoterapia (11-13).

El sistema Allred scoring system (ALLRED) es un instrumento clínico que combina el porcentaje de células marcadas para el receptor y la intensidad de la tinción para evaluar la positividad de los ER y PR (tabla 1), se define un resultado francamente negativo si el score es menor de 2 y positivo si pasa este valor. Se pueden presentar casos en que tumores con menos del $1.0 \%$ de tinción obtienen un score final superior a 2 dado que se acompañan de una intensidad intermedia o fuerte siendo denominados como positivos para marcadores hormonales, según lo menciona la $\mathrm{CAP} / \mathrm{ASCO}$ estos resultados no son comunes y se carece de estudios que demuestren su respuesta o falla en el manejo con hormonoterapia (11-13). Aun así estos valores de referencia son aceptados y aplicados en la práctica clínica diaria.

Para la medición de los receptores HER2 se pueden usar tres pruebas principalmente, estas son: inmunohistoquímica (IHC), la fluorescencia de hibridación in situ (FISH) y tecnología cromogénica de sonda de sustracción de hibridación in situ (SPoT-Light CISH) (14, 15). La prueba de IHC es la más frecuentemente usada; esta consiste en fijar una muestra de tejido embebido en parafina utilizando formol tamponado al $10 \%$ por un periodo de 6 a 48 horas (12); posteriormente se realiza la detección del receptor HER2 en la membrana celular por medio de anticuerpos, este es el blanco de la terapia especifica antiHER2 y por lo tanto la sobreexpresión de esta proteína predeciría la respuesta a dicho agente; son muchas las variables que pueden ocasionar falsos negativos o positivos como el almacenamiento prolongado, la recuperación antigénica, el tipo de anticuerpo utilizado, el sistema de medición y la variabilidad del análisis, por eso la importancia de que los criterios de interpretación e informe de resultados estén estandarizados $(12,13,15)$. Las pruebas de inmunohistoquímica son reportadas de la siguiente manera: 0 o $1+$ equivale a un resultado negativo (Figura 3 ), $2+$ es un resultado limítrofe (Figura 4) y un valor de $3+$ es considerado un resultado positivo $(6,12,15,16)$. (Figura 5).

\section{Epidemiologia de los fenotipos del cáncer de mama}

En la literatura se describe que los carcinomas de mama de tipo luminal son los más frecuentes $(6,7,16-19)$. En $2010 \mathrm{se}$ evaluaron doce estudios con una población de 10,159 casos de cáncer de mama, de los cuales $78.0 \%$ de los tumores corresponden al tipo luminal (7), y entre ellos el más frecuente corresponde a los de tipo luminal A. 


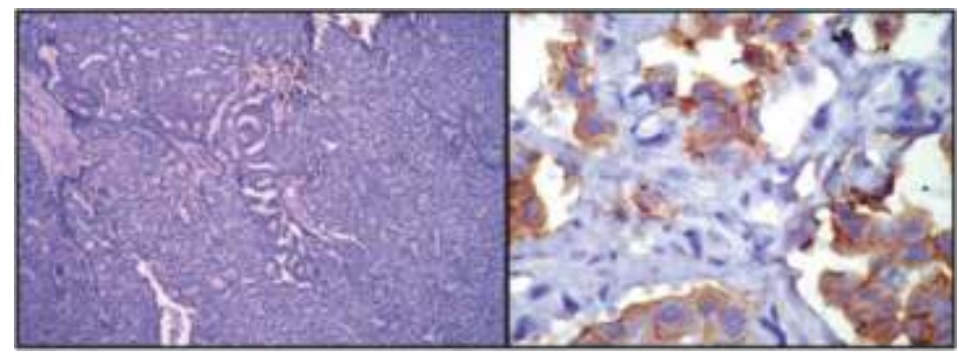

Fuente: Fabiola Navidad Cervera, Miguel A Chávez Martínez; Trascendencia de la determinación del Her2 por inmunohistoquímica y su utilidad terapéutica; Patología Revista latinoamericana Volumen 50, núm. 2, abril-junio, 2012.

Figura 3. Tinción de inmunohistoquímica para el receptor para el factor de crecimiento de tipo epidérmico humano "HER2" negativo.

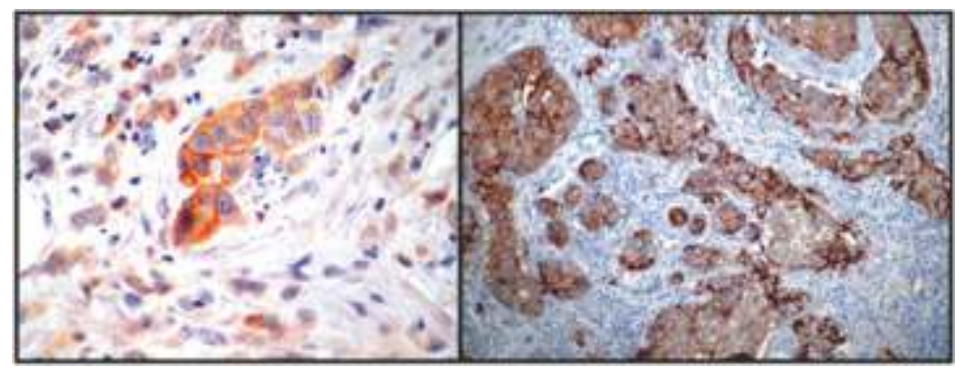

Fuente: Fabiola Navidad Cervera, Miguel A Chávez Martínez; Trascendencia de la determinación del Her2 por inmunohistoquímica y su utilidad terapéutica; Patología Revista latinoamericana Volumen 50, núm. 2, abril-junio, 2012.

Figura 4: Tinción de inmunohistoquímica para el receptor para el factor de crecimiento de tipo epidérmico humano "HER2" intermedio.

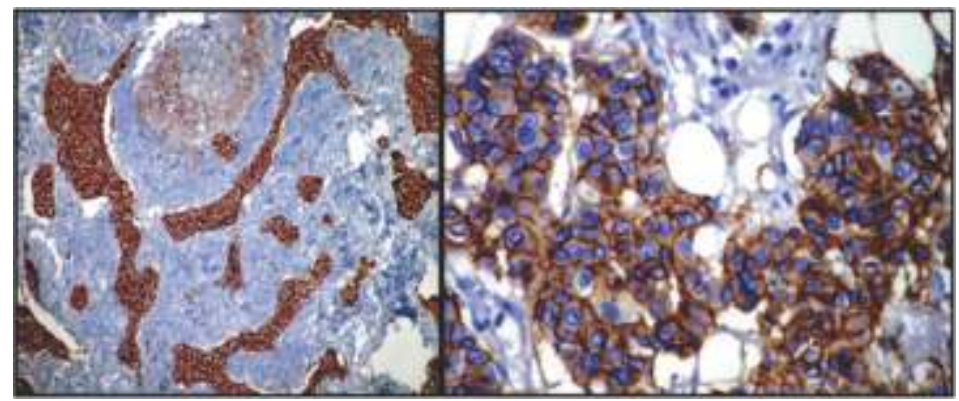

Fuente: Fabiola Navidad Cervera, Miguel A Chávez Martínez; Trascendencia de la determinación del Her2 por inmunohistoquímica y su utilidad terapéutica; Patología Revista latinoamericana Volumen 50, núm. 2, abril-junio, 2012.

Figura 5: Tinción de inmunohistoquímica para el receptor para el factor de crecimiento de tipo epidérmico humano "HER2" positivo.

Hallazgo similar fue encontrado en México durante el 2008, donde el $67.0 \%$ de la población presentó esta clasificación (20). Un estudio realizado en Navarra en el 2011 con 272 pacientes reportó resultados comparables con los referidos en 2010 en el metanálisis antes mencionado, para los tumores luminales $(80.5 \%)$, tumores tipo luminal A $(77.6 \%)$ y luminal B (22.3\%) (6), estadísticas que indican la mayor frecuencia los carcinomas luminal A $(6,17-19,21,22)$.

A los tumores de tipo no luminal, a pesar de compartir como característica la carencia de receptores hormonales, se les han confirmado comportamientos clínicos diferentes entre sí $(20,23)$. Los tumores categorizados en el grupo HER2 positivos son un grupo de tumores que no se han visto asociados a factores de riesgo como la raza, la edad u otros reconocidos (23). Un estudio realizado en Carolina del Norte en el año 2000 reporta que el $20.0 \%$ de los casos estudiados (577) fueron positivos para el marcador HER2 (24). Más recientemente, en 2010, se informó que de 632 casos solo el 6.9\% correspondieron al grupo HER2 (7); siendo este valor cercano a la proporción $(9.9 \%)$ de tumores HER2 positivos reportados en 2011 en España (6). 
A diferencia de los tumores HER 2 positivo, los tumores pertenecientes al subgrupo triple negativo se han visto relacionados con la presencia del gen BRCA $1(6,23)$. En estudios realizados en los años 2008, 2010 y 2011 se informó una prevalencia de tumores triple negativos que varía entre un $11.9 \%$ hasta un $25.0 \%(7,25,26)$; observándose variación importante según el grupo racial y el estado de menopausia (27). Estudios en los que se contempla la raza, determinaron prevalencia de tumores TNP que varía entre $9.8 \%$ en población principalmente caucásica en España (6), y $23.1 \%$ en población nativa hispana en México (25). Al comparar la relación entre el estado de menopausia y la ascendencia afroamericana se encontró en mujeres no afroamericanas indiferente del estado de pre o post menopausia, una frecuencia similar de presentación de los tumores CBP (16.0\%), frente a un $39.0 \%$ y $14.0 \%$ en pacientes afroamericanas pre menopáusicas y afroamericanas posmenopáusicas respectivamente (28). En Colombia, durante el período 2006-2008 se analizaron 1,922 casos, de los cuales 154 eran tumores TNP, cuya prevalencia fue del $8.0 \%$ (27), siendo este valor inferior a lo reportado en la literatura latinoamericana (México 23.1\% y Brasil 75\%) $(25,29)$; se discute si estas diferencias encontradas corresponden a variaciones y mezclas raciales en Colombia pues nuestra descendencia es principalmente caucásica, indoamericana y africana.

\section{Enfoque terapéutico en base a la clasificación inmunohistoquímica del cáncer de mama}

En relación al manejo, entre los años 1970 y 2000, los ensayos clínicos que evaluaron el tratamiento del cáncer de mama estaban fundamentados principalmente en el uso individual o mezcla de quimioterapéuticos como antraciclina, fluorouracilo, epirrubicina, doxorrubicina y ciclofosfamida, aunque estos estudios no contemplaron el perfil inmunohistoquímico de las lesiones tumorales (30). A partir del año 2000, el advenimiento de la terapia con taxanos y los resultados de las investigaciones epidemiológicas contribuyeron al actual enfoque y manejo de cáncer de mama orientado según la clasificación inmunohistoquímica (30). El análisis desde la perspectiva clínica de los marcadores de membrana genera agrupaciones que permiten un uso con mayor significancia para la clasificación inmunohistoquímica, que en el entorno asistencial representa un impacto real y robusto en el pronóstico y en la predicción de la respuesta al tratamiento; así, en la práctica clínica se habla de los TNP que carecen de marcación celular, los tumores con receptor hormonal negativo y positivo para HER2, los que tienen receptor hormonal y HER2 positivo (Luminal B - HER2 Positivo), y un último grupo en el cual persiste la incertidumbre sobre el tratamiento óptimo, son cánceres de mama con receptor hormonal positivos y negativos para HER2, es decir los pertenecientes al espectro luminal (A, indeterminado y $\mathrm{B}$ -
HER2 negativo), entre los cuales se busca establecer la modalidad y patrón correcto para el uso de la quimioterapia (Tabla 2) (31,32).

En las pacientes que presentan tumores del espectro luminal, la opción terapéutica inicial es la hormonoterapia, inhibidores de la aromatasa y los taxanos $(6,20,30)$. Se ha observado que estos tumores tienen baja respuesta al manejo con quimioterapia neoadyuvante o preoperatoria, es decir el uso de agentes quimioterapéuticos con el fin de reducir el volumen tumoral para realizar una intervención quirúrgica definitiva $(6,30)$. Estudios efectuados con el objeto de evaluar la relevancia clínica de la clasificación del cáncer de mama basado en la expresión de marcadores celulares, evidenciaron que los tumores de mama del grupo luminal tienen una respuesta patológica completa a quimioterapia preoperatoria de tan solo el $6.0 \%$ lo que indica una baja respuesta (33). Mientras que el tamoxifeno ha mostrado importantes beneficios en mujeres con tumores mamarios y receptores hormonales positivos (30, $34,35)$; se ha confirmado su eficacia con una reducción de la tasa anual de mortalidad por cáncer de mama de $31.0 \%$ independiente del tipo de quimioterapia o edad, confirmándose su utilidad en mujeres pre menopáusicas con diez años de seguimiento $(34,36)$. El perfil de los tumores luminales implica diferencias en el pronóstico, selección del tratamiento y eficacia del mismo, de este modo encontramos en el espectro luminal a los tumores del grupo A, los catalogados como indeterminados y aquellos que son claramente del grupo $\mathrm{B}$; dicha diferenciación determina cuándo usar quimioterapia, y brinda benéficos específicamente en los casos luminal B en los cuales determinar la presencia del factor Ki-67 aporta a la clínica un alto valor predictivo sobre los beneficios al adicionar esquemas con quimioterapia citotóxica al estándar terapéutico hormonal $(31,32,37-39)$. El Ki-67 es un marcador para la proliferación celular que a mayor expresión se asocia generalmente con un grado más alto y un comportamiento más agresivo, se define la presencia positiva de Ki-67 cuando la expresión es del 20\%, medidas superiores al $30 \%$ son valores claramente altos, y menores del $10 \%$ claramente bajos $(31,32)$.

Por el contrario, los tumores no luminales tienen mayor respuesta patológica completa a los agentes quimioterapéuticos $(30,33)$. En el caso de los TNP no hay un régimen que se considere superior a otro y se ha visto que tienen poca respuesta a las terapias más eficaces con que se dispone para el tratamiento del cáncer de mama (30). Se encontró que las neoplasias TNP expresan buena respuesta a la quimioterapia adyuvante o postquirúrgica con el fin de eliminar células cancerígenas que persisten en el organismo en el postoperatorio, viéndose con regímenes de antraciclina importantes resultados en cuanto a reducción de riesgo de recidiva y mortalidad, e incremento de supervivencia (40). En tumores en estadios tempranos el manejo con quimioterapia citotóxica combinada puede consolidar el plan terapéutico para las neoplasias de este fenotipo, ya sea que se administre en esquema metronómico, es decir, de 
Tabla 2. Características de los grupos clínicos de la clasificación inmunohistoquímica del cáncer de mama.

\begin{tabular}{|c|c|c|c|c|}
\hline \multicolumn{3}{|c|}{$\begin{array}{l}\text { RECEPTOR DE } \\
\text { MEMBRANA }\end{array}$} & \multirow[t]{2}{*}{ GRUPO CLÍNICO } & \multirow[t]{2}{*}{ NOTA } \\
\hline ER & PR & HER2 & & \\
\hline - & - & - & $\begin{array}{l}\text { Tumores triple } \\
\text { negativos }\end{array}$ & $\begin{array}{l}\text { Responden a quimioterapia citotóxica, se pueden considerar } \\
\text { moléculas a base de platino si se identifica mutación del BRCA }\end{array}$ \\
\hline - & - & + & $\begin{array}{c}\text { Receptor hormonal } \\
\text { negativo/HER2 } \\
\text { positivo }\end{array}$ & $\begin{array}{l}\text { Según el estadio puede no ser necesaria terapia sistémica, hasta } \\
\text { indicarse el uso de Quimioterapia más trastuzumab }\end{array}$ \\
\hline $\begin{array}{l}+ \\
0 \\
-\end{array}$ & $\begin{array}{l}+ \\
0 \\
-\end{array}$ & + & $\begin{array}{l}\text { Receptor hormonal } \\
\text { positivo/HER2 } \\
\text { positivo* } \\
\text { (Luminal B - HER2 } \\
\text { positivo) }\end{array}$ & $\begin{array}{c}\text { Presenta receptores de estrógeno o de progesterona positivos, } \\
\text { Según el estadio puede no ser necesaria terapia sistémica, hasta } \\
\text { indicarse el uso de Quimioterapia más trastuzumab y adicionando } \\
\text { terapia endocrina apropiada. }\end{array}$ \\
\hline \multirow{4}{*}{$\begin{array}{l}+ \\
0 \\
-\end{array}$} & \multirow{4}{*}{$\begin{array}{l}+ \\
0 \\
-\end{array}$} & \multirow{4}{*}{$\cdot$} & $\begin{array}{l}\text { Receptor hormonal } \\
\text { positivo/HER2 } \\
\text { negativo* (Tumores } \\
\text { del Espectro } \\
\text { Luninal) }\end{array}$ & $\begin{array}{l}\text { Presenta receptores de estrógeno o de progesterona positivos } \\
\text { mayor o igual a } 1 \% \text {. Se indica endocrinoterapia según el estado de } \\
\text { menopausia asociada o no a quimioterapia según se estime la } \\
\text { respuesta. }\end{array}$ \\
\hline & & & - Luminal A & $\begin{array}{c}\text { Tienen pronóstico favorable. Alta marcación de ER/PR, con bajo Ki- } \\
67 \text {, bajo o ausente afectación ganglionar (N 0-3) y tamaño pequeño } \\
\text { T (T1 T2). Se indica solo endocrinoterapia según el estado de } \\
\text { menopausia. }\end{array}$ \\
\hline & & & - Indeterminado & $\begin{array}{l}\text { Persiste la incertidumbre sobre el grado de riesgo y capacidad de } \\
\text { respuesta a las terapias endocrinas y citotóxicas. }\end{array}$ \\
\hline & & & $\begin{array}{l}\text { - Luminal B (HER2 } \\
\text { negativo) }\end{array}$ & $\begin{array}{c}\text { Baja marcación de ER/PR, con alto Ki-67, extensa afectación } \\
\text { ganglionar, grado histológico 3, extensa invasión linfovascular, un } \\
\text { mayor tamaño T (T3). Se indica endocrinoterapia según el estado } \\
\text { de menopausia asociada a quimioterapia citotóxica. }\end{array}$ \\
\hline
\end{tabular}

Fuente: Tomado y adaptado de: Coates AS, Winer EP, Goldhirsch A, Gelber RD, Gnant M, Piccart-Gebhart M, et all. Tailoring therapies-improving the management of early breast cancer: St Gallen International Expert Consensus on the Primary Therapy of Early Breast Cancer 2015. Annals of Oncology 2015; 26: 1533-1546.

ER: receptores de estrógeno, PR: receptores de progesterona positivos, HER2: receptor para el factor de crecimiento de tipo epidérmico humano, Ki-67: marcador de proliferación celular, implicado en la determinación de la respuesta y los patrones de uso de quimioterapia en los tumores del espectro luninal. -: negativo, +: positivo, + o -: positivo o negativo, * Debe ser positivo para al menos uno de los 2 marcadores hormonales (ER o PR).

forma más frecuente, y a dosis inferiores mínimamente tóxicas dirigidas al endotelio o estroma tumoral; o a dosis densa haciendo referencia a concentraciones con potencial citotóxico completo dirigida a las células neoplásicas, administrada de forma más seguida que en un plan de tratamiento estándar de quimioterapia (41). En la actualidad se está considerando el uso de moléculas con base en platino como cisplatino y carboplatino que actúan uniéndose con el ADN, ARN y otras macromoléculas inhibiendo la replicación, transcripción y síntesis de proteínas desencadenando apoptosis celular (30,31); estos son medicamentos interesantes para el manejo de los TNP, pero aún no se cuenta con indicaciones específicas para añadirlos a los estándares de tratamiento fuera del entorno de un ensayo clínico (30).
Para el manejo de los cánceres de mama positivos para HER2 además de la quimioterapia $(30,31,33)$, se cuenta con agentes blancomoleculares como el trastuzumab $(6,20$, $30,31)$; ya se ha probado que este anticuerpo monoclonal anti-HER2 es eficaz y seguro en el manejo neoadyuvante, adyuvante y en la enfermedad metastásica del cáncer de mama $(6,30,31)$; se ha demostrado una significativa mejoría en la supervivencia libre de enfermedad y en la supervivencia global cuando este es utilizado junto a la quimioterapia $(30,31,42-44)$, y en caso de identificarse el grupo clínico HER2-positivo/ER o PR positivos se debe agregar al manejo el esquema de endocrinoterapia indicado según su estado de menopausia (31). De forma regional en la Fundación Santa Fe de Bogotá se realizó un estudio que evaluó la respuesta del trastuzumab en diversos escenarios 
de cáncer de mama con sobreexpresión del receptor HER2 y se encontró que las pacientes tratadas con quimioterapia más trastuzumab presentaron mejores resultados en el tiempo a la progresión, con mejor tasa de respuesta global y además que el $47.0 \%$ de los casos con compromiso metastásico visceral tuvieron regresión completa de los síntomas asociados (45).

\section{Pronóstico según el estadio y el tipo histológico partiendo de la clasificación inmunohistoquímica}

La literatura es enfática en afirmar que los carcinomas mamarios del subtipo luminal tienen buen pronóstico en comparación con los tumores de los subtipos no luminal (6, 7, 9, 20,31). Frecuentemente, los subtipos luminal A tienen mejor pronóstico que los luminal $\mathrm{B}(7,9,31)$. Un estudio realizado en España reportó que las pacientes que presentaron tumores luminal A mostraron mayor supervivencia y menor riesgo de recaída comparado con los tumores luminal B; igualmente en este estudio se concluye que los cánceres de mama CBP y HER2 positivo tienen peores características clínico-patológicas e inmunohistoquímicas (6); estos hallazgos coinciden con otros estudios $(24,46-48)$.

En relación al tamaño tumoral, las neoplasias mamarias de tipo luminal presentan un tamaño más pequeño $(<2.0 \mathrm{~cm})$ al momento del diagnóstico $(6,7,47)$. A diferencia de los tumores HER2 positivos y TNP que suelen debutar con tamaño superior $(>2.0 \mathrm{~cm})(7,47)$. Los tumores de tipo luminal suelen asociarse a menor invasión ganglionar en comparación con los tumores no luminales. Trabajos realizados en los Estados Unidos describen que en la mayoría de los casos los tumores de tipo luminal no muestran compromiso ganglionar, predominando este comportamiento en las formas que carecen del marcador HER2 (7). Resultados comparables a lo descrito en España (6); donde adicionalmente se reporta que los tumores de tipo HER2 positivos la mayoría (40.7\%) se expresan en estadio pN1 (metástasis en 1 a 3 ganglios linfáticos axilares y/o en ganglios de la mamaria interna), y los tumores TNP del subtipo CBP el 54.6\% presenta compromiso ganglionar (6). Se ha visto en la literatura que los tumores del grupo basal nuclear a pesar de ser frecuentemente de alto grado, no guardan relación con la invasión ganglionar $(28,49,50)$, el cual es uno de los factores pronósticos más importante del cáncer de mama (6).

Se reporta una asociación entre la clasificación inmunohistoquímica del cáncer de mama con los grados histopatológicos; los carcinomas mamarios de tipo luminal suelen asociarse con un bajo a moderado grado histológico, mientras que los carcinomas mamarios de tipo HER2 positivo y TNP se presentan más frecuentemente en grados histológicos avanzados $(6,7,17-19,21,28)$.

\section{Conclusiones}

La clasificación inmunohistoquímica del cáncer de mama tiene claro impacto en el contexto clínico en términos de diagnóstico, pronóstico y tratamiento. Esta ofrece alternativas que sobrepasan otras formas de clasificación fundamentadas en la tipificación y diferenciación morfológica celular, entregando herramientas para comprender con mayor claridad los perfiles de expresión, comportamiento clínico y factores pronósticos. El establecimiento de los grupos clínicos basados en la clasificación inmunohistoquímica ofrece al profesional sanitario la posibilidad de realizar un diagnóstico certero e indicar una terapéutica oncológica específica basada en predicciones sobre la capacidad de respuesta del tumor al tratamiento y de esa manera se brinda un manejo integral e individual con alto impacto en el pronóstico y especialmente evitando tratamientos que podrían ser excesivos y/o insuficientes.

\section{Conflicto de intereses}

Los autores manifiestan que no tienen ningún conflicto de interés.

\section{Referencias}

1. Globocan 2012, Estimated cancer Incidence, Mortality, Prevalence and Disability-adjusted life years (DALYs) Worldwide in 2012. International agency for research on cancer.

2. Organization pan Americana de la salud. Cancer de mama en las Americas. Washington. D.C: paho.org. 2014 [actualización el miércoles 07 de octubre de 2015; acceso el sábado 12 de Octubre de 2015]. Disponible en: http://www.paho.org/hq/index.php?option=com content\& view=category\&layout=blog\&id=3400\&ltemid=3639\&lang =es

3. Robles S, Galanis E. Breast cancer in Latin America and the Caribbean. Rev Panam Salud Pública /Pan Am J Public Health 200211 (3): 178-185.

4. Uribe CJ, Osma SE, Herrera V. Cancer incidence and mortality in the Bucaramanga metropolitan area, 20032007.Colomb méd 2012; 43 (4):290-297.

5. Osma SE, Uribe CJ. Tasas de incidencia y mortalidad por cáncer de mama en el área metropolitana de Bucaramanga en el período 2001-2005. MedUNAB 2014; 16(3):101-111.

6. Arrechea M, García V, Córdoba A, Ibáñez B, Santamaría M, Guillén F. Subtipos moleculares del cáncer de mama: implicaciones pronósticas y características clínicas e inmunohistoquímicas; An. Sist. Sanit. Navar. 2011; 34 (3):219-233.

7. Blows F, Driver K, Schmidt M, Broeks A, Leeuwen F. Subtyping of Breast Cancer by Immunohistochemistry to Investigate a Relationship between Subtype and Short and Long Term Survival: A Collaborative Analysis of Data for 10,159 Cases from 12 Studies. PloS Med 2010; 7 (5): e1000279. 
8. Tang P, Skinner KA, Hicks DG. Molecular Classification of Breast Carcinomas by Immunohistochemical Analysis, Are We Ready?. Diagn Mol Pathol 2009;18(3): 125-132.

9. Sorlie T, Tibshirani R, Parker J, Hastie T, Marron J, Nobel A, et al. Repeated observation of breast tumor subtypes in independent gene expression data sets. Proc Natl Acad Sci USA 2003; 100 (14):8418-8423.

10. Sorlie T, Perou C, Tibshirani R, Aas T, Geisler S, Johnsen $\mathrm{H}$ et al. Gene expression patterns of breast carcinomas distinguish tumor subclasses with clinical implications. Proc Natl Acad Sci U SA 2001; 98 (19): 10869-10874.

11. Hammond MEH, Hayes DF, Dowsett M, Allred DC, Hagerty KL, Badve S; et al. American Society of Clinical Oncology/College of American Pathologists Guideline Recommendations for Immunohistochemical Testing of Estrogen and Progesterone Receptors in Breast Cancer (Unabridged Version). Arch Pathol Lab Med. 2010; 134: 907-922.

12. Fitzgibbons P, Deborah D, Alsabeh R, Berman M, Hayes $D$, Hicks D, et al. Template for Reporting Results of Biomarker Testing of Specimens From Patients With Carcinoma of the Breast. College of American Pathologists 2014; 138: 595-601.

13. Lester S, Bose S, Chen Y, Connolly J, De Baca M, Fitzgibbons $\mathrm{P}$, et al. Protocol for the Examination of Specimens From Patients With Invasive Carcinoma of the Breast, Protocol applies to all invasive carcinomas of the breast, including ductal carcinoma in situ (DCIS) with microinvasion; Based on AJCC/UICC TNM, 7th edition; 2012.

14. Eptein M, Ma Y, Press M. ERBB2 Testing: Assessment of Status for Targeted Therapies. En: Harrys J et al. Disease of the breast. 4ta edition. Philadelphia: Lippincott Williams \& wilkins, a wolters Kluwer business; 2010.

15. Wolff AC, Hammond ME, Hicks DG, Dowsett $M$, McShane LM, Allisonet $\mathrm{KH}$, et al. Recommendations for human epidermal growth factor receptor 2 testing in breast cancer: American Society of Clinical Oncology/College of American Pathologists clinical practice guideline update. $J$ Clin Oncol 2013; 31 (31): 3997-4013.

16. Cervera F, Chávez M. Trascendencia de la determinación del Her2 por inmunohistoquímica y su utilidad terapéutica. Revista latinoamericana Patología 2012; 50 (2):87-93.

17. Carey L, Claire E, Sawyer L, Gatti L, Moore D, Collichio F, Ollila D, Sartor Graham M, Perou C. The Triple Negative Paradox: Primary Tumor Chemosensitivity of Breast Cancer Subtypes. Clin Cancer Res 2007; 13(8) 23292334.

18. Bodour S, Coya T, Ishak E, Gaber S, Berguis B, Hussain K, Duquette R, Resau J, Carpten J. Molecular subtype analysis determines the association of advanced breast cancer in Egypt with favorable biology. BMC Women's Health 2011; 11:1-9.

19. Tamimi D, Shawarby M, Ahmed A, Hassan A, Alodaini A. Protein expression profile and prevalence pattern of the molecular classes of breast cancer - a Saudi population based study. BMC cáncer 2010; 10:1-13.

20. Zepeda E, Recinos E, Cuellar M, Robles C, Maffs E. Clasificación molecular del cáncer de mama. Rev. Cirugía y Cirujanos.2008; 76 (1): 87-93.

21. Calderón A, Martínez G, Gallardo A, Rojas A, Flórez C. Triple Marker Immunohistochemestry Analysis in Breast Cancer Mexican Patients. Patología Revista latinoamericana 2012; .50 (2): 72-79.

22. Su Y, Zheng Y, Zheng W, Gu K, Chen Z, Li G, et al; Distinct distribution and prognostic significance of molecular subtypes of breast cancer in Chinese women: a population-based cohort study. BMC Cancer 2011; 11:111.

23. Carey L, Perou C, Dressler L, Livasy C, Geradts J, Cowan $\mathrm{D}$, et al. Race and the poor prognosis basal-like breast cancer (BBC) phenotype in the population-based Carolina Breast Cancer Study. J Clin Oncol 2004; 22 (14): 9510.

24. Huang W, Newman B, Millikan R, Conway K, Hulka B, Schell M, Liu E. Risk of breast cancer according to the status of HER-2/neu oncogen amplification. Cáncer Epidemiol Biomarkers Prev 2000; 9: 65-71.

25. Lara-Medina F, Pérez-Sánchez V, Saavedra-Pérez D, et al. Triple-negative breast cancer in hispanic patients: High prevalence, poor prognosis, and association with menopausal status, body mass index, and parity. Cancer. 2011;117: 3658-69.

26. Liedtke $\mathrm{C}$, Mazouni $\mathrm{C}$, Kenneth $\mathrm{R}$, André $\mathrm{H}$, Tordai $\mathrm{A}$, Mejia J, et al. Response to Neoadjuvant theraphy and long-term survival. Journal clinical Oncology 2008; 26: 1275-1281.

27. García O, Ossa C, Beltrán M, Cano M, Villamizar L, Arias A. Descripción de una cohorte de pacientes con cáncer de mama triple-negativo subtipo basal-like, atendidas en el Instituto Nacional de Cancerología y en el Hospital de San José durante el periodo 2006-2008. Rev Colomb Cancerol 2012. 16 (2): 91-99.

28. Carey L, Perou C, Livasy C, Dressler L, Cowan D, Conway $\mathrm{K}$ et al. Race, breast cancer subtypes, and survival in the Carolina Breast Cancer Study. JAMA 2006; 295 (21): 2492-2502.

29. De Brot M, Soares FA, Stiepcich M, et al. Basal-like breast cancers: clinicopathological features and outcome. Rev Assoc Med Bras. 2009; 55: 529-34.

30. National Cancer Institute. Breast Cancer Treatment $(P D Q \circledR)$ - Health Professional Version. United States of America: PDQ Adult Treatment Editorial Board; 2016; $\mathrm{D}$ i s p o n i b l e e n : http://www.cancer.gov/types/breast/hp/breast-treatmentpdq.

31. Coates AS, Winer EP, Goldhirsch A, Gelber RD, Gnant M, Piccart-Gebhart M, et all. Tailoring therapies-improving the management of early breast cancer: St Gallen International Expert Consensus on the Primary Therapy of Early Breast Cancer 2015. Annals of Oncology 2015; 26: 1533-1546

32. Tang $P$, Tse GM. Immunohistochemical Surrogates for Molecular Classification of Breast Carcinoma, A 2015 Update. Arch Pathol Lab Med 2016; 140.

33. Rouzier R, Perou CM, Symmans WF, et al. Breast cancer molecular subtypes respond differently to pre-operative chemotherapy. Clin Cancer Res. 2005; 11: 5678-5685.

34. Early Breast Cancer Trialists' Collaborative Group (EBCTCG): Effects of chemotherapy and hormonal therapy for early breast cancer on recurrence and 15-year survival: an overview of the randomised trials. Lancet 2005; 365 (9472): 1687-717.

35. Colleoni M, Gelber S, Goldhirsch A, et al.: Tamoxifen after adjuvant chemotherapy for premenopausal women with lymph node-positive breast cancer: International Breast Cancer Study Group Trial 13-93. J Clin Oncol 2006; 24 (9): 1332-41.

36. Davies C, Pan H, Godwin J, et al.: Long-term effects of continuing adjuvant tamoxifen to 10 years versus stopping at 5 years after diagnosis of oestrogen receptor-positive 
breast cancer: ATLAS, a randomised trial. Lancet 2013; 381 (9869): 805-16.

37. de Azambuja E, Cardoso F, de Castro G, Jr et al. Ki-67 as prognostic marker in early breast cancer: a meta-analysis of published studies involving 12155 patients. Br J Cancer 2007; 96: 1504-1513.

38. Sinn P, Aulmann S, Wirtz R, Schott S, Marmé F, Varga Z, et al. Multigene Assays for Classification, Prognosis, and Prediction in Breast Cancer: a Critical Review on the Background and Clinical Utility. Geburtshilfe Frauenheilkd 2013; 73(9): 932-940.

39. Criscitiello C, Disalvatore D, De Laurentiis M et al. High Ki67 score is indicative of a greater benefit from adjuvant chemotherapy when added to endocrine therapy in luminal B HER2 negative and node-positive breast cancer. Breast 2014; 23: 69-75.

40. EarlyBreast CancerTrialists'Collaborative Group(EBCTCG). Peto R, Davies C, Godwin J, et al:: Comparisons between different polychemotherapy regimens for early breast cancer: meta-analyses of longterm outcome among 100,000 women in 123 randomised trials. Lancet 2012; 379 (9814): 432-44.

41. Mehta RS: Dose-dense and/or metronomic schedules of specific chemotherapies consolidate the chemosensitivity of triple-negative breast cancer: a step toward reversing triple-negative paradox. J Clin Oncol 2008; 26 (19): 32868; author reply 3288.

42. Perez E, Romond E, Suman V, et al.: Updated results of the combined analysis of NCCTG N9831 and NSABP B31 adjuvant chemotherapy with/without trastuzumab in patiens with HER2-positive breast cancer. J Clin Oncol 2007; 25 (Suppl 18): 512, 6s.
43. Romond EH, Perez EA, Bryant J, et al.: Trastuzumab plus adjuvant chemotherapy for operable HER2-positive breast cancer. N Engl J Med 2005; 353 (16): 1673-84.

44. Slamon D, Eiermann W, Robert N, et al.: Adjuvant trastuzumab in HER2-positive breast cancer. N Engl J Med 2011; 365 (14): 1273-83.

45. Carranza H, Vargas C, Cardona A, Yepes A, Ospina V, Otero $\mathrm{E}$, et al. Tratamiento con trastuzumab en diversos escenarios del cáncer de mama: experiencia en la Fundación Santa Fe de Bogotá. Rev Colomb Cancerol 2007; 11(1):23-31.

46. Kreike B, Kouwenhove V, Horlings $H$, Weigelt B, Peterse $\mathrm{H}$, Bartelink $\mathrm{H}$ et al. Gene expression profiling and histopathological characterization of triple-negative/basallike breast carcinomas. Breast Cancer Res. 2007; 9 (5): R65.

47. Uribe JR, Hernández CA, Menolascino F, Rodríguez JE, Istúriz LM, Márquez ME. Clasificación molecular del cáncer de mama y su correlación clínica. Rev Venez Oncol 2010; 22(2): 109-116.

48. Vallejos CS, Gómez HL, Cruz WR, et al. Breast cancer classification according to immunohistochemistry markers: subtypes and association with clinicopathologic variables in a peruvian hospital database. Clinical Breast Cancer. 2010;10:294-300.

49. Rakha EA, Putti TC, Abd El-Rehim DM Paish C, Green $A R$, Powe DG, Lee AH et al. Morphological and immunophenotypic analysis of breast carcinomas with basal and myoepithelial differentiation. J Pathol 2006; 208: 495-506.

50. Silva L, Clarke C, Lakhani S. Demystifying basal-like breast carcinomas. J Clin Pathol 2007; 60 (12): 1328-1332. 